\title{
IMPLICANCIAS SOCIOEDUCATIVAS DE LA GENERATIVIDAD EN EDUCADORES RURALES CHILENOS
}

\author{
Eduardo Sandoval Obando \\ Psicólogo \\ eduardo.sandoval.o@gmail.com
}

\begin{abstract}
Recepción Artículo: 27 enero 2021
Admisión Evaluación: 27 enero 2021

Informe Evaluador 1: 29 enero 2021

Informe Evaluador 2: 31 enero 2021

Aprobación Publicación: 1 febrero 2021
\end{abstract}

Trabajo financiado por la Agencia Nacional de Investigación y Desarrollo (ANID) / FONDECYT DE INICIACIÓN № 11190028 "La Profesionalidad Docente Rural: Implicaciones Socioeducativas desde la Perspectiva Narrativa Generativa" (2019-2022).

\section{RESUMEN}

La generatividad se transforma en una dimensión educativa prometedora entre quienes ejercen la profesionalidad docente rural en contexto escolares chilenos. Su desarrollo estaría dado por el conjunto de comportamientos, prácticas y estrategias que orientan el quehacer profesional del educador rural, manifestándose en los saberes, experiencias y dinámicas relacionales que establecen con el alumnado y sus comunidades de origen, favoreciendo el reconocimiento histórico-cultural de las tradiciones y características particulares del territorio en el que se encuentra la escuela rural. Objetivo: interpretar y comprender, desde una perspectiva narrativa generativa, las pautas de comportamiento construidas por el profesorado rural, contribuyendo a la sistematización y generación de nuevas formas de comprensión del quehacer profesional docente rural. Método: se adopta un enfoque interpretativo cualitativo, siguiendo un diseño descriptivo, exploratorio y transversal. La muestra es de carácter intencional, conformada por 6 educadores que poseen al menos 30 años de experiencia en escuelas rurales presentes en las Regiones Metropolitana, La Araucanía y los Ríos (Chile). Para la interpretación de los datos, se recurre al análisis de contenido, siguiendo la lógica de la Teoría Fundamentada y las Entrevistas en Profundidad desde la perspectiva narrativa generativa. Resultados: Preliminarmente, las historias de vida de los educadores rurales evidencian el desarrollo de prácticas pedagógicas potencialmente generativas, caracterizadas por la autonomía, humildad y el optimismo con el que se relacionan cotidianamente con el alumnado y sus familias, destacándose la implicación pedagógica y las altas expectativas frente al desarrollo de sus estudiantes como dimensiones de enorme valor educativo en la ruralidad chilena.

Palabras Claves: generatividad; historias de vida; implicación pedagógica; profesionalidad docente rural 


\section{ABSTRACT}

Socio-educational implications of generativity in chilean rural educators. Generativity becomes a promising educational dimension among those who exercise rural teaching professionalism in Chilean school contexts. Its development would be given by the set of behaviors, practices and strategies that guide the professional work of the rural educator, manifesting itself in the knowledge, experiences and relational dynamics that they establish with the students and their communities of origin, favoring the historical-cultural recognition of the traditions and particular characteristics of the territory in which the rural school is located. Objective: to interpret and understand, from a generative narrative perspective, the behavior patterns built by rural teachers, contributing to the systematization and generation of new ways of understanding the rural professional teaching task. Method: a qualitative interpretive approach is adopted, following a descriptive, exploratory and cross-sectional design. The sample is intentional, made up of 6 educators who have at least 30 years of experience in rural schools present in the Metropolitan, La Araucanía and Los Ríos Regions (Chile). For the interpretation of the data, content analysis is used, following the logic of grounded theory and in-depth interviews from the generative narrative perspective. Results: In a preliminary way, the life stories of rural educators show the development of potentially generative pedagogical practices, characterized by the autonomy, humility and optimism with which they interact daily with students and their families, highlighting the pedagogical implication and the high expectations regarding development. of its students as dimensions of enormous educational value in Chilean rural education.

Keywords: generativity; life histories; pedagogical involvement; rural teaching professionalism.

\section{INTRODUCCIÓN}

La generatividad, caracterizada como la expresión equilibrada de tendencias ligadas al desarroIlo personal y al cuidado de los otros (Erikson, 2000), cultivando un legado que perdure en el tiempo (McAdams y De St. Aubin, 1992; Villar, López y Celdrán, 2013; Sandoval-Obando y Zacarés, 2020), resulta particularmente significativa en el ejercicio pedagógico, desplegado en las escuelas rurales chilenas ${ }^{1}$ en donde el profesorado está llamado a ser responsable de otros. Su importancia estaría dada por el conjunto de acciones, prácticas y estrategias que caracterizan las historias de vida construidas por el profesorado en estos contextos (Sandoval-Obando, 2020), develando aqueIlas pautas de comportamiento que le otorgan una identidad narrativa positiva e integradora acerca de las experiencias acumuladas a lo largo de la vida (McAdams y Olson, 2010; Balfour, 2012). En lo pedagógico, sería observable en los objetivos que se proponen, las clases y dinámicas relacionales que establecen con el alumnado (Sánchez, 2001; Arias e Iglesias, 2015), los saberes que promueven e incluso el enfoque educativo al cual se adscriben (Vera, Osses y Schiefelbein, 2012).

El Proyecto FONDECYT de Iniciación № 11190028 (Sandoval-Obando, 2019) invita a preguntarse lo siguiente: ¿Cómo se forma personal y pedagógicamente, un educador/a para desempeñarse en contextos de ruralidad? ¿Qué reflexiones, saberes y criterios de acción pedagógica emergen a partir de las trayectorias vitales del profesorado rural respecto al funcionamiento de la institución escolar? ¿Cuáles son las dinámicas relacionales, que el profesorado rural ha construido a lo largo de sus trayectorias vitales? ¿Articula el profesorado acciones y prácticas generativas que enriquecen el proceso de enseñanza y aprendizaje en contextos de ruralidad? Nuestro propósito es interpretar y comprender, desde una perspectiva narrativa generativa, las pautas de comportamiento construidas por el profesorado rural, contribuyendo a la sistematización y generación de nuevas formas de comprensión del quehacer profesional docente rural. 


\section{MÉTODO}

\section{Diseño del Estudio}

La investigación se construye desde un enfoque interpretativo cualitativo, a partir de un diseño descriptivo, exploratorio y transversal. Como técnica de recolección de datos se recurre a las Entrevistas en Profundidad (Kvale, 2011), desde la perspectiva narrativa generativa (Serra, 2008; McAdams y McLean, 2013; Sandoval-Obando, 2020). Específicamente, se profundiza en las trayectorias vitales (personales y pedagógicas) de los y las educadores/as rurales que transitan desde la adultez media a la vejez, en donde es posible observar la emergencia de acciones y comportamientos potencialmente generativos con los que asumen cotidianamente los procesos de enseñanza y aprendizaje en la ruralidad chilena.

\section{Participantes}

Se utiliza una muestra intencional, en concordancia con lo señalado por Otzen y Manterola (2017) puesto que las personas se eligen en función del grado en que se ajustan a los criterios 0 atributos establecidos para el estudio. Así, la lógica de muestreo apunta a la diversidad de casos posibles (Flick, 2015), por lo que durante cada año de ejecución de la investigación, se trabaja con 6 educadores/as (2 por cada región), que se hayan desempeñado pedagógicamente en el contexto rural chileno por al menos 30 años, que residan en alguna de las regiones donde se centra el estudio (Metropolitana, La Araucanía y Los Ríos) y que estén vivenciando la transición de la adultez media a la tardía. En vista de lo anterior, la muestra quedó conformada para el primer año de ejecución del estudio, por 4 hombres (2 educadores de la Región de La Araucanía y 2 de la Región de Los Ríos, respectivamente) y 2 mujeres (pertenecientes a la región Metropolitana), cuya edad promedio es de 60,5 años; Ios que en su conjunto acumulan un total promedio de 34,5 años de experiencia profesional docente en contextos de ruralidad.

\section{La Perspectiva Narrativa Generativa: Un Campo de Posibilidades para la Investigación Socioeducativa}

Este enfoque metodológico permite ahondar en las experiencias del profesorado rural, explorando 'desde abajo y desde dentro' lo que ocurre en la cotidianeidad de la vida rural, develando íntimamente a los/as educadores/as y mirando el mundo desde sus propios ojos como herramientas cualitativas para la obtención de datos (Sandoval-Obando, 2014).

En lo procedimental y de acuerdo con lo planteado desde el FONDECYT № 11190028 (SandovalObando, 2019), las Historias de Vidas desde la perspectiva narrativa generativa (McAdams, 2008), se construyen a partir de los relatos obtenidos mediante el uso de las Entrevistas de Historias de Vida (McAdams, 2015). Para cumplir con lo anterior, se ha contemplado la realización de 3 entrevistas, separadas entre sí por un periodo de tiempo previamente acordado con cada uno de los participantes. En el primer encuentro, se le solicita al participante que describa y profundice en los principales acontecimientos que han marcado su vida y que, de una u otra forma, estén relacionados con cambios significativos en su vida 0 en los de su entorno. Este primer momento, permite caracterizar el proceso de construcción del 'Yo Narrativo' (McAdams y Olson, 2010), brindando elementos valiosos para la comprensión de la personalidad del participante y el nivel de autoconocimiento alcanzado, las etapas y periodos críticos experimentados como educadores/as y que le han permitido comprender, desde una perspectiva histórico cultural, la escuela rural, tal como la percibe hoy.

En el siguiente encuentro, se le pregunta al sujeto acerca de los vacíos identificados, así como por los recuerdos más significativos que hayan surgido como consecuencia del primer encuentro 
hasta el momento actual, avanzando en la identificación de los puntos de giro, en los que existe un punto de inflexión en el curso vital del participante. Es decir, episodios clave que marcan un cambio importante en la historia de vida (McAdams, 2008), y los que, de una u otra forma, favorecen la capacidad de 'darse cuenta' sobre las implicancias y significados de estos sucesos en su vida.

En un tercer momento, y tras realizar el proceso de codificación y transformación de los datos que han surgido en las primeras entrevistas, se profundiza en la identificación y caracterización de aquellos hitos relacionados con acciones y prácticas generativas, relacionado con la identidad narrativa (McAdams y Olson, 2010). Esta dimensión permite dos ámbitos potenciales de análisis: el interno, referido al modo en que los esfuerzos, acciones y prácticas generativas o no, se integran en la historia de vida del participante, y el externo, entendido como aquel que posibilita la comparación de los relatos con el resto de los participantes, observándose diferencias en el desarrollo generativo alcanzado (McAdams y Logan, 2004). Además, se ahonda en las demandas culturales percibidas por los sujetos en su rol docente (es decir, cómo y qué tipo de responsabilidades han asumido a lo largo de sus vidas), las conductas y prácticas que reflejen el interés o preocupación consciente por las próximas generaciones y cómo se expresa en los procesos de enseñanza y aprendizaje al interior de la escuela rural, la presencia de metas a futuro que orienten su proyecto de vida y que tengan impacto en el contexto educativo e histórico-cultural en el que se desenvuelven como educadores/as.

\section{Estrategia de Análisis y Producción de la Información}

Para la interpretación y producción de los datos, se recurre al análisis de contenido, siguiendo la lógica de la Teoría Fundamentada (Strauss y Corbin, 2002). Así, los datos recopilados, una vez transcritos, se sometieron a un proceso de análisis riguroso, asistido por computador con el apoyo de la herramienta informática NVivo 12.0. Además, el protocolo de investigación se construyó de acuerdo con las directrices establecidas por el Comité de Ética Científico para el trabajo con seres humanos de la Universidad Autónoma de Chile (Chile).

\section{RESULTADOS}

En esta sección, se describen algunos de los resultados preliminares obtenidos durante el primer semestre de ejecución del FONDECYT № 11190028 (Sandoval-Obando, 2019). Específicamente, se presentan algunas de las categorías teóricas - conceptuales emergentes con sus respectivas citas representativas, otorgando voz y sentido a las historias de vida del profesorado que dan vida a esta investigación.

En primer lugar, se observa que el profesorado valora la escuela rural como un espacio-tiempo que posibilita el despliegue de la autonomía en su quehacer docente, brindándoles mayores grados de libertad en la organización de los saberes y actividades que orientan cotidianamente su práctica pedagógica en la ruralidad chilena. Lo anterior, les otorga un mayor grado de autoeficacia sobre su quehacer docente, lo que se traduce en una mayor confianza con el alumnado, así como una relación marcada por el afecto positivo y la valoración de sus experiencias previas. Al respecto, los/as participantes señalan que:

“... Bueno, primero que todo, aquí (en el ámbito rura)l se trabaja con más tranquilidad, con más autonomía, se pueden tomar mejores decisiones, 10 otro, el grupo es pequeño, por lo tanto es fácil cohesionarlo, gente joven (refiriéndose a los estudiantes) que a veces puede tener dificultades personales, a veces por conducta, eso es lo que nos interesa... nos interesa que aporten y si no tienen aporte..., aquí está (refiriéndose a la escuela) lo que tienen que adquirir en su conocimiento..." (Alejandro, Docente Rural, Región de La Araucanía).

En segundo lugar, se observa que el profesorado rural manifiesta una trayectoria vital (personal 
y pedagógica) caracterizada por una apertura permanente y desafiante frente a su propio proceso de aprendizaje. Es decir, emerge el autodesarrollo como una dimensión característica de la generatividad, que se manifiesta en la formación permanente del profesorado para afrontar los diversos desafíos y demandas expresadas por el alumnado rural, posibilitando la transmisión de saberes coherentes con los requerimientos e intereses del alumnado dentro de los entornos educativos en los que se desenvuelven:

"...me ha tocado compartir con colegas rurales casi de todo Chile y también efectuar pasantías... hemos hecho pasantías con los colegas en el que he gestionado yo, donde hemos salido a visitar otros centros rurales... otras realidades, en una ocasión como microcentro viajamos también a Argentina, a un intercambio con profesores rurales de Argentina, de Neuquén... también lo hemos hecho con las colegas de Lonquimay, de Galvarino, por el caso de nuestra escuela también con el pueblo de Empedrado... he tenido una grata satisfacción de haber tenido esos logros..." (Alvaro, Educador Rural, Región de La Araucanía).

En tercer lugar, los/as participantes manifiestan un compromiso profundo y genuino hacia la profesión docente, influido por sus propias experiencias vitales relacionadas con la enseñanza en la ruralidad chilena. Lo anterior, les permite mantener altas expectativas frente al desarrollo y posibilidades educativas del alumnado, independientemente de sus condiciones de origen. Al respecto, Javiera (Educadora Rural, Región Metropolitana) expresa con claridad el interés genuino de aportar al desarrollo de sus estudiantes mediante su quehacer profesional docente:

"...Yo creo que lo primero que tienen que tener es altas expectativas con estos niños, porque fuera de que ya están ellos en un entorno tan pobre, imagínese que yo le digo a estos mismos niños de la... que están a cuantas kilómetros de Santiago, no conocían el metro, cuando fuimos también a Constitución... una niña iba mirando todo... porque todo le llamaba la atención... entonces el profesor tiene que ser creativo... tener hartas expectativas de los niños, no ponerles cartel, de que porque está en la población tanto, este no va a llegar a ninguna parte, no, uno nunca sabe lo que hay detrás de... ¿la tapa del torero es el dicho?, puede salir un profesional mejor que uno, nadie sabe lo que hay detrás de cada persona, yo siempre les digo, usted haga cuenta que están enseñándoles a sus hijos, ustedes no van a mandar a sus hijos a una escuela a que vaya a jugar, eso tienen que hacer cuando hacen la clase ustedes, yo no tengo hijos, pero les enseño como si fueran mis sobrinos...".

En cuarto lugar, el profesorado rural entrevistado manifiesta un marcado optimismo hacia su quehacer profesional docente, mostrándose orgullosos/as por las experiencias acumuladas a lo largo de su trayectoria vital. Además, se vería reflejado en el entusiasmo, alta satisfacción y motivación con la que afrontan cotidianamente los procesos de enseñanza y aprendizaje desplegados en la ruralidad chilena:

“...cualquier trabajo que uno haga, por más humilde que sea... tiene que hacerlo bien... digamos hacerlo con alegría, hacerlo con vocación, tiene que hacerlo de la mejor manera, sea lo que sea... gracias a Dios que tengo una profesión y una profesión que la llevo con alegría..." (Carlos, Educador Rural, Región de Los Ríos).

“...el pensamiento positivo, mirar las cosas con fe, con esperanza con amor... Hay que ser un poco idealista, buscar lo mejor de todo... me orienta el mismo pensamiento... ese de La Vida es Bella... porque es como una utopía, que los chicos traten de estar siempre alegres contentos, a pesar de que hay esta situación de riesgo o de peligro... nos permite conversar más..." (Manuel, Educador Rural, Región de Los Ríos).

Por último, se podría inferir que el potencial generativo manifestado por los/as educadores/as rurales entrevistados/as caracteriza la implicación pedagógica, caracterizado como aquel conjunto 
de estrategias, acciones y herramientas desplegadas por los docentes para construir un espaciotiempo educativo propicio para el desarrollo de aprendizajes significativos, relevantes y pertinentes en su alumnado, mediante la vinculación afectiva y recíproca entre profesor / estudiante, el autoreconocimiento de los errores y el interés por transmitir experiencias y aprendizajes prácticos coherentes con sus experiencias vitales:

“...ya van 38 años y considero que es muy importante mi información, tanto personal, familiar, social (refiriéndose a su propia experiencia vital)... me ha marcado como persona en lo familiar (con el nacimiento de mis hijos)... y en lo social, en cada comunidad que estuve, los sellos de cada comunidad me marcaban... tuve que involucrarme en las actividades de cada sector, tanto de la parte deportiva, como en lo social también con ellos... han sido experiencias riquísimas que me han favorecido en mi relación con mi familia y también con mis colegas educadores... con la junta de vecinos tenemos una relación muy cercana... porque nuestra escuela es el centro de actividades del sector rural donde se encuentra... En lo pedagogico, con mis alumnos trato de estar ahí... estar involucrado con ellos, de saber sus limitaciones, sus potencialidades, de poder escucharlos, también de la familia, de ir a la casa, de poder saber su forma de vida, para así poder aconsejar y poder también ayudar en la medida de mis medios, poder hacer un labor que pueda ayudar en la vida de mis alumnos...". (Manuel, Educador Rural, Región de Los Ríos).

\section{DISCUSIÓN}

Los relatos del profesorado rural permiten visibilizar comportamientos potencialmente generativos de enorme riqueza para la cultura docente rural (Fernández, 2010). Específicamente, el potencial generativo se manifestaría tempranamente en la trayectoria vital (personal y pedagógica) de los/as participantes, permitiéndoles asumir su quehacer docente con autonomía y libertad, transformándola en una profesión eminentemente generativa, en donde confluirían al menos 3 dimensiones: afecto positivo, satisfacción vital y satisfacción laboral (Ackerman, Zuroff y Moskowitz, 2000).

Del mismo modo, las categorías emergentes evidencian que los docentes rurales manifestarían una expresión generativa que es coherente y consistente con su rol laboral (Zacarés, Ruiz y Amer, 2002; Sandoval-Obando, 2020), propiciando una implicación activa en la tarea docente para aprovechar las oportunidades de desarrollo, formación y enriquecimiento pedagógico que le brinda la escuela rural chilena así como valoración histórica-cultural de las costumbres y saberes presentes en el territorio. Lo anterior, es coherente con lo señalado por Zeichner, Bowman, Guillen y Napolitan (2016) quienes enfatizan la importancia de que los maestros conozcan las comunidades en las que se desenvuelven sus estudiantes, propiciando la construcción de dinámicas relacionales respetuosas y de confianza con las familias del alumnado rural así como con los diversos actores claves presentes en el territorio, integrando los saberes y costumbres populares dentro del proceso de aprendizaje de sus estudiantes.

En cuanto a las expectativas que los y las entrevistados/as manifiestan frente a las posibilidades de desarrollo de sus estudiantes, la información recopilada es coherente con lo reportado por Díaz, Osses y Muñoz (2016) quienes identificaron que los docentes rurales chilenos tienden a construir (consciente e inconscientemente) una práctica docente optimista y flexible, permitiéndoles generar lazos afectivos que promueven y refuerzan el aprendizaje, otorgándole mayor sentido de autoeficacia a su práctica pedagógica. Por ende, el afecto positivo (cercanía, humildad y compromiso docente genuino con su labor educativa) beneficiaría el proceso de aprendizaje (Villarroel y Leiva, 2005; Sandoval-Obando, 2017; Vera, 2020). Incluso, a pesar de no contar con la formación o las competencias necesarias para un trabajo psicosocial y/o comunitario, son conscientes del importante rol 
que cumplen en su comunidad educativa, asumiendo el desafío de superar las condiciones de vulnerabilidad y/o precariedad que caracteriza el entorno próximo de sus estudiantes, fijándose objetivos prosociales (Son y Wilson, 2011) desafiantes para la superación personal y movilidad social del alumnado rural.

Por consiguiente, pareciera ser que este tipo de vínculos y de prácticas potencialmente generativas exhibidas por el profesorado rural (visitas domiciliarias, actividades deportivas y caminatas, inclusión de las juntas de vecinos y organizaciones sociales presentes en el territorio, etc.), serían coherente con investigaciones en donde se observa que la implicación pedagógica con enfoque comunitario incidiría positivamente en la formación y desarrollo del profesorado rural en diversas dimensiones tales como: incentivaría la reflexión crítica y la toma conciencia acerca de la naturaleza de su función en dichos espacios y tiempos educativos, potenciaría el reconocimiento y valoración de la diversidad cultural en el aula, reconocería las fortalezas y potencialidades de las diversas familias y comunidades que conforman la escuela, fortalecería la confianza y las habilidades para relacionarse pedagógicamente con las familias y los miembros de la comunidad (Vesely, Brown y Mehta, 2017).

Del mismo modo, el optimismo pedagógico que caracteriza los relatos aportados por los participantes describen la visión de vida futura que posee cada educador/a rural hacia sus estudiantes, contraponiéndose con las expectativas del estudiante y/o de su grupo familiar de origen, quienes en muchos casos arrastran trayectorias escolares marcadas por la pobreza, la cesantía y las condiciones de vulnerabilidad educativa, los que directa 0 indirectamente operan como factores determinantes en el proceso de enseñanza y aprendizaje (Cornejo y Redondo, 2007). Por lo tanto, las altas expectativas del profesorado rural y el optimismo que caracteriza su quehacer docente, adquiere importancia desde la perspectiva narrativa generativa, ya que en la mayoría de los casos, sus experiencias vitales marcadas por el esfuerzo (retrospección de su propia vida), la perseverancia, el compromiso y el interés genuino por aportar al desarrollo de las generaciones más jóvenes, sentaría las bases para la configuración de una práctica docente potencialmente generativa, en concordancia con lo descrito por Mizala, González, Romaguera y Guzmán (2000) al identificar que las dificultades superadas por el/la docente, le otorgan un insumo importante para transmitir sus experiencias y saberes a los/as niños/as, particularmente cuando los profesores rurales provienen de estratos sociales bajos, principalmente de clase media, obrera y campesina.

\section{CONCLUSIONES}

A modo de conclusión, este trabajo de investigación en curso (Sandoval-Obando, 2019) aborda una línea de investigación cualitativamente profunda y enriquecedora en torno al potencial generativo observado en la profesionalidad docente rural chilena, develando la implicación pedagógica, el optimismo y las altas expectativas frente a las posibilidades de desarrollo del alumnado, como dimensiones educativas relevantes para el análisis y comprensión de la práctica docente rural. Lo anterior, es posible mediante la sistematización y valoración de aquellas experiencias personales y profesionales acumuladas por los/as docentes rurales a lo largo de sus trayectorias vitales, en complemento con las desafiantes y heterogéneas vicisitudes que surgen durante la transición de la adultez media a la vejez (An y Cooney, 2006, Taylor, 2006; Wojciechowska, 2017), constituyéndose en un grupo de interés y de enorme valor para la mejora de la educación rural en lo particular, y la educación en general durante la compleja realidad socio-sanitaria actual.

Finalmente, este trabajo devela la importancia del trabajo docente como figura activa en la vida social-comunitaria que rodea la escuela e incentiva la reflexión crítica acerca del proceso de formación y desarrollo socio-histórico del profesorado rural chileno en donde el estudio de la generativi- 
dad permitiría visibilizar algunos criterios de acción pedagógica relevantes para la transformación y mejora de los procesos de enseñanza y aprendizaje (Liu y Ball, 2019), convirtiéndose en una alternativa educativa relevante para la promoción de un aprendizaje sentido y con sentido para nuestros niños/as y jóvenes.

\section{REFERENCIAS BIBLIOGRÁFICAS}

Ackerman, S., Zuroff, D. y Moskowitz, D.S. (2000). Generativity in midlife and young adults: links to agency, communion, and subjective well-being. International Journal of Aging and Human Development, 50(1), 17-41.

An, J. y Cooney, T. (2006). Psychological well-being in mid to late life: The role of generativity development and parent-child relationships across the lifespan. International Journal of Behavioral Development, 30(5), 410-421. Doi: https://doi.org/10.1177/0165025406071489

Arias, A. y Iglesias, S. (2015). La Generatividad como una Forma de Envejecimiento Exitoso. Estudio del efecto Mediacional de los Vínculos Sociales. European Journal of Investigation in Health, Psychology and Education, 5(1), 109-120.

Balfour, J. (2012). Rurality research and rural education: Exploratory and explanatory power. Perspectives in Education, 30(1),9-18.

Cornejo, R. y Redondo, J. (2007). Variables y factores asociados al aprendizaje escolar. Una discusión desde la investigación actual. Estudios Pedagógicos, 33(2), 155-175.

Díaz, R., Osses, S. y Muñoz, S. (2016). Factores e interacciones del proceso de enseñanza-aprendizaje en contextos rurales de la Araucanía, Chile. Estudios Pedagógicos (Valdivia), 42(3), 111128.

Erikson, E. (2000). El Ciclo Vital Completado. Barcelona: Paidós

Fernández, B. (2010). Un Análisis Multidimensional del Síndrome de Burnout en Profesorado de Conservatorios y Enseñanza Secundaria. Tesis de Doctorado en Psicología, Dpto. de Psicología Evolutiva y de la Educación, Universidad de Valencia. Recuperado de http://hdl.handle.net/10803/78805

Flick, U. (2015). El diseño de investigación cualitativa. Madrid: Morata

Kvale, S. (2011). Las Entrevistas en Investigación Cualitativa. Madrid: Morata.

Liu, K. y Ball, A. (2019). Critical Reflection and Generativity: Toward a Framework of Transformative Teacher Education for Diverse Learners. Review of Research in Education, 43(1), 68-105. Doi: https://doi.org/10.3102/0091732X18822806

McAdams, D. (2015). Tracing Three Lines of Personality Development. Research in Human Development, 12, 224-228. https://doi.org/10.1080/15427609.2015.1068057

McAdams, D. y McLean, K. (2013). Narrative identity. Current Directions in Psychological Science, 22(3) 233-238. Doi: https://doi.org/10.1177/0963721413475622

McAdams, D. (2013). The Positive Psychology of Adult Generativity: Caring for the next generation and constructing a Redemptive Life. En J. Sinnott (ed.), Positive Psychology: Advances in understanding adult motivation (pp. 191-205). New York: Springer.

McAdams, D. y Olson, B. (2010). Personality Development: Continuity and Change Over the Life Course. Annual Review of Psychology, 61, 517-542. Doi: 10.1146/annurev.psych.093008.100507

McAdams, D. (2008). The Life Story Interview. Evanston: The Foley Center for the Study of Lives / Northwestern University. Recuperado de https://www.sesp.northwestern.edu/foley/instruments/interview/

McAdams, D. y Logan, R. (2004). What is generativity? En De St. Aubin, E.; Mc.Adams, D. y Kim, T. The Generative Society: Caring for Future Generations (pp. 15-31). Washington, DC: APA. 
McAdams, D. y De St. Aubin, E. (1992). A theory of generativity and its assessment trough selfreport, behavioral acts, and narrative themes in autobiography. Journal of Personality and Social Psychology, 62, 1003-1015

Mizala, A., González, P., Romaguera, P. y Guzmán, A. (2000). Los Maestros en Chile: Carreras e Incentivos. Washington, D.C.: Banco Interamericano de Desarrollo.

Moreno, C. (2007). Las Escuelas Rurales en Chile: La Municipalización y sus fortalezas y debilidades. Revista Digital eRURAL, Educación, cultura y desarrollo rural, 4(8). Recuperado de http://www.scielo.org.co/scielo.php?script=sci_nlinks\&ref=000149\&pid=S1657$9267201400020001800019 \& \operatorname{lng}=\mathrm{pt}$

Otzen, T. y Manterola, C. (2017). Técnicas de Muestreo sobre una Población a Estudio. International Journal of Morphology, 35(1), 227-232. Doi: https://dx.doi.org/10.4067/S071795022017000100037

Peirano, C.; Puni, S. y Astorga, M. (2015). Educación Rural: Oportunidades para la Innovación. Cuadernos de Investigación Educativa, 6(1), 53-70.

Sánchez, T. (2001). Creencias acerca del proceso educativo en alumnas de la carrera de Educación de Párvulos: Universidad Católica de Temuco. Tesis para optar al grado de Magíster en Estudios Psicológicos. Temuco: Universidad de La Frontera.

Sandoval-Obando, E. (2020). Caracterizando la Identidad Narrativa en Educadores Rurales Chilenos: Una Propuesta de Investigación. En Sandoval-Obando, E., Serra Desfilis, E. y García, O. Nuevas Miradas en Psicología del Ciclo Vital (pp. 273-298). Santiago de Chile: RIL Editores / Universidad Autónoma de Chile. Doi: https://doi.org/10.32457/ISBN9789568454951982020ED1

Sandoval-Obando, E. (2019). La Profesionalidad Docente Rural: Implicaciones Socioeducativas desde la Perspectiva Narrativa Generativa. FONDECYT de Iniciación № 11190028. Temuco: Documento sin publicar.

Sandoval-Obando, E. (2017). El Docente como Mediador Emocional y Cognitivo de Jóvenes en Contextos Vulnerados: Tensiones y Desafíos para la Transformación de la Práctica Pedagógica. Tesis conducente al Grado Doctor en Ciencias Humanas. Facultad de Filosofía y Humanidades, Universidad Austral de Chile. Recuperado de: http://cybertesis.uach.cl/tesis/uach/2017/egs218d/doc/egs218d.pdf

Sandoval-Obando, E. (2014). Propensión a Aprender de los Adolescentes Infractores de Ley: Reflexiones desde el Enfoque Biográfico. Polis, 13 (37), 251-273.

Sandoval-Obando, E. y Zacarés, J. (2020). Generatividad y Desarrollo Adulto. En Sandoval-Obando, E., Serra Desfilis, E. y García, 0. Nuevas Miradas en Psicología del Ciclo Vital (pp. 189-218). Santiago de Chile: RIL Editores / Universidad Autónoma de Chile. Doi: https://doi.org/10.32457/ISBN9789568454951982020-ED1

Serra, E. (2008). Somos lo que contamos: La Historia de Vida como método evolutivo. En F. Sanz, La fotobiografía: imágenes e historias del pasado para vivir con plenitud el presente (pp. 405415). Barcelona: Kairós.

Son, J. y Wilson, J. (2011). Generativity and volunteering. Sociological Forum, 26(3), 644-667. Recuperado de 10.1111/j.1573-7861.2011.01266.x.

Strauss, A. y Corbin, J. (2002). Bases de la Investigación Cualitativa. Técnicas y Procedimientos para Desarrollar la Teoría Fundamentada. Antioquia: Universidad de Antioquia.

Taylor, A. (2006). Generativity and Adult Development: Implications for Mobilizing Volunteers in Support of youth. En J. Rhodes y E. Clary (Eds.), Mobilizing adults for positive youth development: Strategies for closing the gap between beliefs and behaviors (pp. 83-100). New York, NY: 
Springer.

Vesely, C., Brown, E. y Mehta, S. (2017). Developing cultural humility through experiential learning: How home visits transform early childhood preservice educators' attitudes for engaging families. Journal od Early Childhood Teacher Education, 38(3), 242-258. Doi: https://doi.org/10.1080/10901027.2017.1345805

Vera, L. (2020). El Saber Pedagógico de Docentes Normalistas. Historias de Vida de Maestras Provenientes de Diferentes Escuelas Normales Superiores. Tesis presentada para optar al Grado de Magíster en Educación. Bogotá: Universidad Militar de Nueva Granada. Recuperado de https://repository.unimilitar.edu.co/handle/10654/35879

Vera, D., Osses, S. y Schiefelbein, E. (2012). Las Creencias de los profesores rurales: una tarea pendiente para la investigación educativa. Estudios pedagógicos (Valdivia), 38(1), 297-310. Doi: https://dx.doi.org/10.4067/S0718-07052012000100018

Villar, F., López, O. y Celdrán, M. (2013). La generatividad en la vejez y su relación con el bienestar: ¿Quién más contribuye es quien más se beneficia? Anales de Psicología, 29(3), 897-906.

Villarroel, G. y Leiva, P. (2005). Emoción y aprendizaje: un estudio en estudiantes de Educación Básica Rural. Revista Digital eRural, Educación, cultura y desarrollo rural, 8(4).

Wojciechowska, L. (2017). Subjectivity and generativity in midlife. Polish Psychological Bulletin, 48(1), 38-43. Doi: https://doi.org/10.1515/ppb-2017-0005

Zacarés, J., Ruiz, J. y Amer, E. (2002). Generatividad y bienestar psicológico en profesores: un estudio exploratorio. En M. I. Fajardo, M. I. Ruiz, A. Ventura, F. Vicente \& J. A. Julve (Comps.), Psicología de la Educación y Formación del profesorado. Nuevos retos, nuevas respuestas (pp. 611-625). Badajoz: Psicoex.

Zeichner, K., Bowman, M., Guillen, L. y Napolitan, K. (2016). Engaging and Working in Solidarity With Local Communities in Preparing the Teachers of Their Children. Journal of Teacher Education, 67(4), 277-290. Doi: https://doi.org/10.1177/0022487116660623

1 De acuerdo a Peirano, Puni y Astorga (2015), la existencia de cursos multigrado es la principal característica de las escuelas rurales, permitiendo que los procesos de enseñanza y aprendizaje se desarrollen dentro de una misma aula, combinando al menos dos niveles, hasta 6o básico (Moreno, 2007). 